\title{
MATHEMATIC MODELING OF A NEW INLAY BUOYANCY ARTEFACT
}

\author{
K. Lehrmann ${ }^{1}$, F. Härtig ${ }^{1}$, R. Tutsch ${ }^{2}$ \\ ${ }^{1}$ Physikalisch-Technische Bundesanstalt, Braunschweig, Germany, katharina.lehrmann@ptb.de \\ 2 Technical University Braunschweig, Institute of Production Metrology, Braunschweig, Germany, r.tutsch@tu-bs.de
}

\begin{abstract}
:
The novel buoyancy artefact concept for the use in mass metrology enables the determination and correction of systematic air buoyancy effects caused by measurements outside a vacuum. In contrast to other buoyancy artefacts with a nominal mass of $1 \mathrm{~kg}$, the new artefact, called inlay artefact, has a smaller volume resulting from an enclosed tungsten core. The theoretical design of the new artefact consists of dismountable discs with spherical distance pieces and a cylinder. This work focuses on the design and mathematical model considering limiting factors caused by the handling and the geometry of the comparators used.
\end{abstract}

Keywords: buoyancy artefact; kilogram; silicon; inlay artefact; revised SI

\section{INTRODUCTION}

Highly accurate mass calibrations require high sophisticated mass comparators which are operated in vacuum. Outside a vacuum the measurements are being strongly influenced by environmental conditions, such as buoyancy effects. This is particularly evident, if mass standards of the same nominal value made of different materials such as steel, platinum-iridium, or silicon are calibrated on the basis of a substitution method or compared against each other. In this case buoyancy effects significantly influence the findings because of the resulting different volumes [1]. One way to correct the buoyancy effects is to determine the air densities by using what is known as buoyancy artefacts calibrated by volume [2]. Most accurate results for the determination of air densities are achievable with a relative uncertainty in the order of magnitude of $5 \times 10^{-5}$ [3]. Typically, aside the same nominal weight such artefacts have nearly the same surface areas and surface properties but different volumes. The greater the volume difference of buoyancy artefacts used, the more reliably the air density can be determined. A design is presented in order to achieve a large volume difference between buoyancy artefacts used.

\section{DESIGN OF A NEW KIND OF BUOYANCY ARTEFACT}

Figure 1 shows a sketch of the proposed new buoyancy artefact. It consists of several discs made of monocrystalline silicon and one cylinder made of silicon with a tungsten core enclosed. The entire standard is referred to as an "inlay artefact". According to the principal approach of buoyancy artefacts, the nominal mass of the artefact $M_{\text {ref }}$ is $1 \mathrm{~kg}$ and its nominal surface area must be identical to the surface area of the other buoyancy artefacts $A_{\text {ref }}$ used. Due to the tungsten core the effective density of the artefact is much higher than the density of the buoyancy artefacts made of pure silicon. This leads to a smaller volume and thus, to less buoyancy. Distance pieces, which are part of a statically determined coupling, allow the stackable and demountable individual parts of the artefact to be dismantled for cleaning purposes.

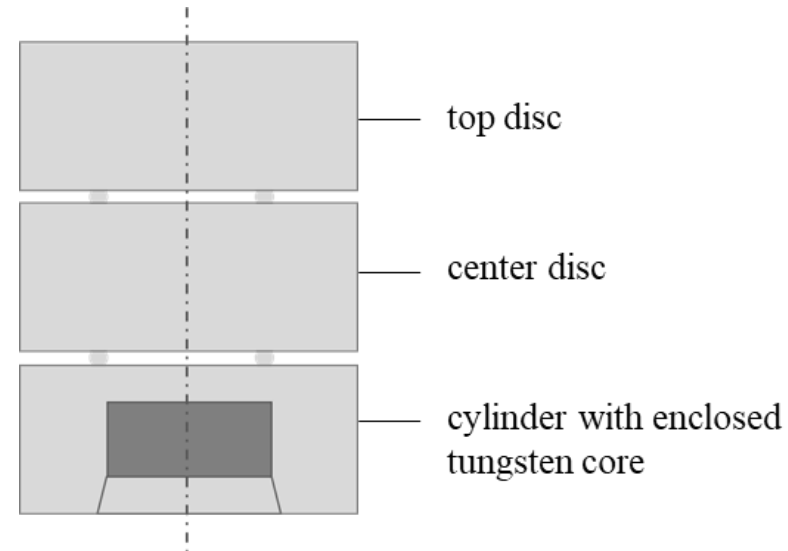

Figure 1: Exemplary figure of an inlay buoyancy artefact with two discs (top and center disc) and a cylinder with enclosed tungsten core

For modeling the new buoyancy artefact, the following variable input parameters apply:

- the surface area ratio between discs and the cylinder

- the number of discs

- the diameter of the discs

- the diameter of the cylinder 
For practical handling the radius of the discs and the cylinder should be equivalent. To achieve the specific surface areas, the surface area ratio of discs to cylinder should be at least two. First investigations have shown that the required surface area can be manufactured by three identical discs made of pure silicon. In order to provide enough volume to include a tungsten core the size of the ground disc have to be enlarged. From now on the ground disc was named cylinder (silicon disc with tungsten core). An additional estimation shows that the resulting surface area could be realized by a minimum of two further discs. Further requirements for the new buoyancy artefact are shown in Table 1 and depend on the geometric specifications of the comparators used, practical handling and the considerations of tilting stability.

Table 1: Settings for numerical iteration

\begin{tabular}{|l|c|}
\hline Parameter & Range \\
\hline max. total height & $h_{\max } \leq 105 \mathrm{~mm}$ \\
\hline tilt stability & $20 \mathrm{~mm} \leq r_{\mathrm{dsc}}, r_{\mathrm{cyl}} \leq 45 \mathrm{~mm}$ \\
\hline number of discs & $2-5$ \\
\hline
\end{tabular}

The silicon cylinder and the plug enclosing the inlay core are detachably bonded to each other according to the specifications of conical tapered ground joints [4] as used for gas-tight silicon oxide compounds.

For all calculations, the density of silicon is assumed to be $2328.8 \mathrm{~kg} / \mathrm{m}^{3}$ [5] and for tungsten is assumed to be $19250.0 \mathrm{~kg} / \mathrm{m}^{3}$ [6].

\section{MATHEMATIC MODELING OF A NEW KIND OF BUOYANCY ARTEFACT}

For the sophisticated calculation of the inlay artefact, a number of limiting conditions have to be considered. For example, the surface area must correspond to a specified reference surface area and at the same time the mass must correspond to a specified nominal mass. In addition to the basic cylindrical geometry, the geometries of the spherical distance pieces and chamfers have to be taken into account for constructive reasons. Based on all these complex and correlated specifications, the radii and heights of the discs and the cylinder must be precisely dimensioned. In this section, first the calculations for the surface areas and then the masses are outlined.

The corrections caused by chamfers are addressed comprehensively in section 4, while corrections relating to distance pieces are explained in section 5. In the following, this refers to all subsequent calculations that correlate with chamfers and distance pieces.

\subsection{Calculation of the Surface Areas for Cylinder and Discs}

The pure surface area $A_{\mathrm{dsc}}$ of all discs, without any corrections for chamfers and distance pieces, depends on the given surface area of the reference $A_{\text {ref }}$, and the surface area ratio of discs to cylinder, denoted ratio. This results in:

$A_{\mathrm{dsc}}=A_{\text {ref }} \frac{\text { ratio }}{\text { ratio }+1}-A_{\text {corr,dsc,cal }}+A_{\text {corr,dsc,cha }}$

and includes the correction for chamfers $A_{\text {corr,dsc,cha }}$, and the correction for distance pieces $A_{\text {corr,dsc,cal }}$. The surface of the cylindrical part is obtained as:

$A_{\text {cyl }}=A_{\text {ref }} \frac{1}{\text { ratio }+1}-A_{\text {corr,cyl,cal }}+A_{\text {corr,cyl,cha }}$

including the reference surface $A_{\text {ref }}$, surface area ratio of discs to cylinder ratio and the correction for distance pieces $A_{\text {corr,cyl,cal }}$ as well as the correction for chamfers $A_{\text {corr,cyl,cha. }}$. The height of a disc $h_{\mathrm{dsc}}$ is calculated from:

$h_{\mathrm{dsc}}=\frac{\frac{A_{\mathrm{dsc}}}{n_{\mathrm{dsc}}}-2 \pi \cdot r_{\mathrm{dsc}}^{2}}{2 \pi \cdot r_{\mathrm{dsc}}}$

with number of discs $n_{\mathrm{dsc}}$ and radius of the disc(s) $r_{\mathrm{dsc}}$. The height $h_{\mathrm{cyl}}$ of a cylinder with radius $r_{\mathrm{cyl}}$ can be calculated as:

$h_{\mathrm{cyl}}=\frac{A_{\mathrm{cyl}}-2 \pi \cdot r_{\mathrm{cyl}}^{2}}{2 \pi \cdot r_{\mathrm{cyl}}}$

with the surface area of the cylinder $A_{\text {cyl }}$ calculated in equation (2).

\subsection{Calculation of Masses of Cylinder and Discs}

The calculation of the total mass shall be composed of the calculation of the mass of the individual discs $M_{\mathrm{dsc}}$ and the mass of the cylinder $M_{\text {cyl }}$. The discs are designed on the basis of the previously determined heights and radii.

The total mass of the cylinder $M_{\text {cyl }}$ consisting of the silicon shell and the inlay core of tungsten is calculated, knowing the reference mass $M_{\text {ref }}$ and the mass of all discs $M_{\mathrm{dsc}}$, by means of:

$M_{\text {cyl }}=M_{\text {ref }}-M_{\mathrm{dsc}}$

The further steps are simplified if only cylindrical geometries are used for calculations. In order to obtain these principles, the masses of the distance pieces and chamfers are extracted from the surrounding silicon cylinder. The pure cylinder mass $M_{\text {cyl,pure }}$ is given by:

$M_{\text {cyl,pure }}=M_{\text {cyl }}-M_{\text {corr,cal }}+M_{\text {corr,cha }}$ 
As previously stated, the corrections for the distance pieces $M_{\text {corr,cal }}$ and chamfers $M_{\text {corr,cha }}$ will be shown in sections 4 and 5 .

Having the volume of the cylinder $V_{\text {cyl,Si,pure }}$, the mass of the tungsten core $M_{\mathrm{cyl}, \mathrm{W}}$, can be described by:

$M_{\mathrm{cyl}, \mathrm{W}}=\frac{M_{\mathrm{cyl}, \mathrm{pure}}-\rho_{\mathrm{Si}} \cdot V_{\mathrm{cyl}, \mathrm{Si}, \mathrm{pure}}}{\rho_{\mathrm{W}}-\rho_{\mathrm{Si}}} \cdot \rho_{\mathrm{W}}$

including the corresponding densities of silicon $\rho_{\mathrm{Si}}$ and tungsten $\rho_{\mathrm{W}}$.

According to:

$V_{\text {cyl,Si,pure }}=V_{\text {cyl,Si }}-V_{\text {corr,cyl,cal }}+V_{\text {corr,cyl,cha }}$

the value of the reduced volume $V_{\text {cyl,Si,pure }}$ of the silicon cylinder considers the volumetric corrections influenced by both, the spherical distance pieces $V_{\text {corr,cyl,cal }}$ and the chamfer $V_{\text {corr,cyl,cha. }}$.

For reasons of symmetry and to simplify the calculation elegantly, the geometric proportions of the enclosing silicon cylinder $r_{\text {cyl }}, h_{\text {cyl }}$ and the internal tungsten core are assumed to be identical, so that:

$\frac{r_{\mathrm{cyl}}}{h_{\mathrm{cyl}}}=\frac{r_{\mathrm{cyl}, \mathrm{W}}}{h_{\mathrm{cyl}, \mathrm{W}}}$

Both the radius $r_{\mathrm{cyl}, \mathrm{W}}$ of the inlay cylinder, given by:

$r_{\text {cyl, } \mathrm{W}}=\sqrt[3]{\frac{V_{\text {cyl, } \mathrm{W}}}{\pi} \frac{r_{\text {cyl }}}{h_{\text {cyl }}}}$

and the height of the inlay cylinder $h_{\mathrm{cyl}, \mathrm{W}}$, given by:

$h_{\mathrm{cyl}, \mathrm{W}}=r_{\mathrm{cyl}, \mathrm{W}} \cdot \frac{r_{\mathrm{cyl}}}{h_{\mathrm{cyl}}}$

are calculated from the fixed ratio of the geometry of the silicon cylinder for reasons of symmetry.

The effective density of the inlay artefact $\rho_{\text {ily }}$ is calculated as:

$\rho_{\text {ily }}=\frac{M_{\mathrm{dsc}}+M_{\mathrm{cyl}, \mathrm{Si}}+M_{\mathrm{cyl}, \mathrm{W}}}{V_{\mathrm{dsc}}+V_{\mathrm{cyl}}}$

derived from the mass of the discs and the mass of the cylinder with the inlay tungsten artefact.

\section{CORRECTION OF CHAMFER}

To correct the chamfers, volume, mass and surface area differences must be determined in comparison with the uncorrected discs and cylinder. The adjustment is classified by cylinder and chamfer.

Figure 2 shows a section through a cylinder or disc with a circumferential chamfer. According to the mathematical description in section 2 , the radii $r_{\mathrm{dsc}}$ or $r_{\text {cyl }}$ must be substituted for the variable $r$. The ring surface $A_{\text {rng }}$, the lateral surface $A_{\text {shl }}$ of the uncorrected cylinder and the resulting chamfer surface $A_{\text {cha }}$ are shown under the chamfer angle $\alpha$.

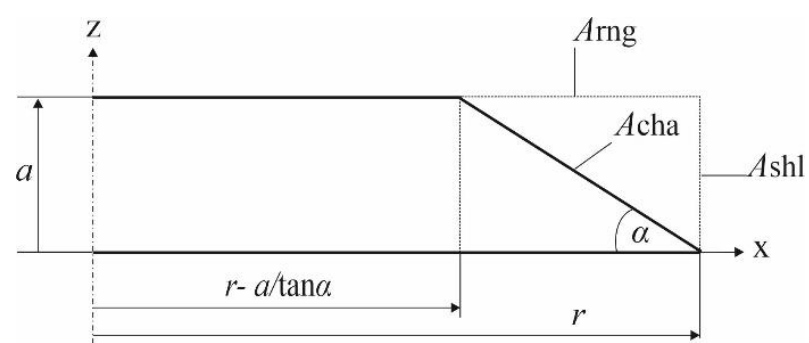

Figure 2: Schematic cross section through a cylindrical object with straight chamfers

\subsection{Calculation of the Surface Areas for Chamfer}

The correction $A_{\text {corr,cha }}$ depends on the surface of the cylinder shell surface area $A_{\mathrm{shl}}$, the ring surface area of the disc $A_{\text {rng }}$ and the surface area of the chamfer $A_{\text {cha }}$. As a result:

$A_{\text {corr,cha }}=\left(A_{\text {rng }}+A_{\text {shl }}-A_{\text {cha }}\right)$.

The surface area of an annular ring of the top face of a disc can be expressed as:

$A_{\text {rng }}=\pi\left(r^{2}-\left(r-\frac{a}{\tan \alpha}\right)^{2}\right)$

The surface area of a disc of solid material without chamfer can be calculated from:

$A_{\mathrm{shl}}=2 \pi \cdot r \cdot a$

For the surface correction of a chamfer the general approach for calculating rotational symmetric bodies [7]:

$A_{\text {cha }}=2 \pi \int_{0}^{a} f(z) \sqrt{1+\left(f^{\prime}(z)\right)^{2}} d z$

is chosen. Herein the function $f(z)$ describes the mathematical cross section of the rotatory body.

$f(z)=r-z \cdot \tan \alpha$

Hence, the correction of the chamfer surface area results in:

$$
A_{\text {cha }}=2 \pi \int_{0}^{a}(r-z \cdot \tan \alpha) \cdot \sqrt{1+(\tan \alpha)^{2}} d z
$$

After resolving the integral, the final correction for all chamfers is:

$$
\begin{aligned}
& A_{\text {corr,cha }}=n_{\mathrm{dsc}} \cdot n_{\mathrm{cha}} \cdot \\
& \left(\begin{array}{c}
-2 \pi \sqrt{1+(\tan \alpha)^{2}} \cdot\left(a \cdot r-\frac{a^{2}}{2}\right)+ \\
2 \pi \cdot r \cdot a+\pi\left(r^{2}-\left(r-\frac{a}{\tan \alpha}\right)^{2}\right)
\end{array}\right)
\end{aligned}
$$




\subsection{Calculation of Volume and Mass of Chamfers}

The mathematical theory results from the approach to calculate a quarter torus. The general formula for the volume of a rotational symmetric object is [7]:

$$
V=\pi \int_{0}^{a}(f(z))^{2} d z
$$

The resulting correction for volume of chamfers is described by the uncorrected cylinder volume $V_{\text {cyl }}$ subtracted by the chamfer volume correction $V_{\text {corr,cha }}$ which results in:

$V_{\text {cha }}=V_{\text {cyl }}-V_{\text {corr,cha }}$

The detailed relation of this correction reads:

$V_{\text {corr,cha }}$

$$
=\pi \int_{0}^{a} r^{2} d z-\pi \int_{0}^{a}(r-z \cdot \tan \alpha)^{2} d z
$$

After solving the integrals, the final volume correction $V_{\text {corr,cha }}$ becomes:

$$
\begin{aligned}
& V_{\text {corr,cha }}=n_{\text {cha }} \cdot \\
& \left(\pi r^{2} a-\pi\left(r^{2} \cdot a+\frac{a^{3}}{3} \cdot(\tan \alpha)^{2}-r \cdot a^{2} \cdot \tan \alpha\right)\right)
\end{aligned}
$$

Assuming the known density $\rho_{\mathrm{Si}}$ and the determined correction $V_{\text {corr,cha }}$, the adjustment for the mass of the chamfers is:

$$
M_{\text {corr,cha }}=\rho_{\mathrm{Si}} \cdot V_{\text {corr,cha }}
$$

\section{CORRECTION OF DISTANCE PIECES}

The distance pieces enlarge the mass and surface area of the entire inlay artefact. Hence, corrective calculations are necessary.

Distance pieces are placed on the top and bottom of the discs and the cylinder in relation to each other as shown in Figure 3. Distance pieces can be attached firmly to a disc in various methods, for example with a bond connection or high-vacuum compatible adhesive.

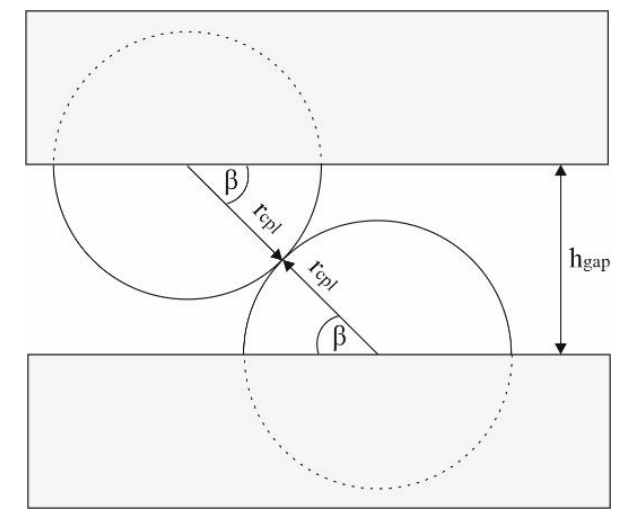

Figure 3: Illustration of gap with spherical distance pieces (silicon spheres, $r=2 \mathrm{~mm}, \beta=45^{\circ}$ )
In this context, only the upper half of the distance pieces must be considered for the correction of mass and surface area.

Table 2 lists the relation between the number of distance pieces and the disc type or cylinder. Good mechanic design points out that a six-point mounting is the most stable stacking method for statically determined bearings. This avoids wiggling or instability of couplings. The selected stacking method requires exactly nine distance pieces per gap. This results in six distance pieces which are fixed on the top side of the cylinder. In addition, for any center disc, there are six distance pieces on the bottom side, and three further distance pieces on the top side. Finally, there are three distance pieces left for the top disc.

Table 2: The total number of distance pieces per top disc, centre disc or cylinder and numbers for variable $n$, where $n$ depends on the number of total discs

\begin{tabular}{|l|c|c|}
\hline $\begin{array}{l}\text { Type of } \\
\text { discs/cylinder }\end{array}$ & $\begin{array}{l}\text { Number of } \\
\text { distance pieces }(\boldsymbol{k})\end{array}$ & $\begin{array}{l}\text { Numbers for } \\
\text { variable }(\boldsymbol{n})\end{array}$ \\
\hline top disc & 3 & 2 \\
\hline center disc(s) & 9 & $n_{\mathrm{dsc}}$ \\
\hline cylinder & 6 & 2 \\
\hline
\end{tabular}

\subsection{Calculation of the Surface Areas for Spherical Distance Pieces}

The surface area correction $A_{\text {corr,cpl }}$ derives from the spherical surface area $A_{\mathrm{cpl}}$. However, the surface area of the cylinder $A_{\text {cir }}$ which is covered by the base of the hemisphere must be subtracted as:

$A_{\text {corr,cpl }}=A_{\text {cpl }}-A_{\text {cir }}$

Using the relevant parameters regarding the number of distance pieces $k$ the correction becomes:

$A_{\text {corr }, \mathrm{cpl}}=\frac{k}{2} \cdot 4 \pi \cdot r_{\mathrm{cpl}}{ }^{2}-\mathrm{k} \cdot \pi \cdot r_{\mathrm{cpl}}^{2}$

\subsection{Calculation of Volume and Mass of Spherical Distance Pieces}

The volume correction through all distance pieces is made considering the number of discs $n$. Polished silicon spheres with a radius of $2 \mathrm{~mm}$ serve as distance pieces.

Depending on the type of disc or cylinder, different numbers of the spherical distance pieces $k$ must be inserted into the calculations. This results in the equation for the volume correction $V_{\text {corr,cpl }}$ according to:

$V_{\text {corr }, \mathrm{cpl}}=(n-1) \cdot \frac{k}{2} \cdot \frac{4}{3} \pi \cdot r_{\mathrm{cpl}}{ }^{3}$

Knowing the density $\rho_{\mathrm{Si}}$ and the calculated correction $V_{\text {corr,cpl }}$ leads to the adjustment for the mass of the distance pieces $M_{\text {corr,cpl }}$ as:

$M_{\text {corr }, \mathrm{cpl}}=\rho_{\mathrm{Si}} \cdot V_{\text {corr,cpl }}$ 


\section{RESULTS}

The parameters for height and radius of the discs and the cylinder are determined numerically by a minimizing fit algorithm (Newtonian method) provided by Mathematica [8]. The solution fulfilling all constraints can be achieved, if discs and the cylinder have an identical diameter and a minimum number of discs is used. This results in a volume difference of approximately $315 \mathrm{~cm}^{3}$ between the new inlay artefact and a silicon buoyancy hollow cylinder with a volume of approximately $646 \mathrm{~cm}^{3}$ [1]. To sum up, 1690 iteration runs were evaluated.

Table 3 shows one solution for the fitted values for the designed inlay artefact, which fulfills all constraints.

Table 3: Model parameters for an inlay artefact

\begin{tabular}{|l|l|l|}
\hline Input parameters & Value & Unit \\
\hline mass & 1 & $\mathrm{~kg}$ \\
\hline ratio & 2 & - \\
\hline radius of disc(s) & 0.034 & $\mathrm{~m}$ \\
\hline radius of cylinder & 0.034 & $\mathrm{~m}$ \\
\hline chamfer angle & 45 & $\circ$ \\
\hline chamfer length & 0.0005 & $\mathrm{~m}$ \\
\hline Fitted parameters & Value & Unit \\
\hline mass of tungsten inlay & 0.26142 & $\mathrm{~kg}$ \\
\hline mass of silicon & 0.73858 & $\mathrm{~kg}$ \\
\hline number of discs & 2 & - \\
\hline total height & 0.0966898 & $\mathrm{~m}$ \\
\hline height of one disc & 0.0301871 & $\mathrm{~m}$ \\
\hline height of cylinder & 0.0306588 & $\mathrm{~m}$ \\
\hline height of tungsten core & 0.0152044 & $\mathrm{~m}$ \\
\hline radius of tungsten core & 0.0168614 & $\mathrm{~m}$ \\
\hline $\begin{array}{l}\text { radial wall thickness } \\
\text { Si cylinder }\end{array}$ & 0.0171386 & $\mathrm{~m}$ \\
\hline $\begin{array}{l}\text { axial wall thickness } \\
\text { Si cylinder }\end{array}$ & 0.0077272 & $\mathrm{~m}$ \\
\hline Results & Value & $\mathrm{Unit}$ \\
\hline volume & 0.00033060 & $\mathrm{~m}{ }^{3}$ \\
\hline $\begin{array}{l}\text { effective density } \\
\text { of new inlay artefact }\end{array}$ & 3024.78 & $\mathrm{~kg} / \mathrm{m}^{3}$ \\
\hline & & \\
\hline
\end{tabular}

\section{SUMMARY}

The aim of this theoretical design study of a new inlay buoyancy artefact was to create a buoyancy artefact of nominal mass $1 \mathrm{~kg}$ with a small volume compared to other buoyancy artefacts. The small volume of the artefact, resulting in a particularly low air buoyancy was achieved by a combination of discs and a cylinder made of silicon, with a tungsten core inside. In contrast to conventional artefacts made of stainless steel, the newly designed artefact can be efficiently and gently cleaned the same way as the reference, like a silicon sphere.

Special focus is given on the correction of chamfer and spherical distance pieces. A version of the inlay artefact is exemplarily calculated and illustrated.

The theoretical design study for parameters, i.e. radius and height of the silicon parts and the tungsten core, were determined by numerous constraints using a variation method and could be a basis for future manufacturing.

\section{REFERENCES}

[1] K. Lehrmann, R. Tutsch, F. Härtig, "Design of sorption and buoyancy artefacts made of silicon", IMEKO, Cavtat-Dubrovnik, 2020, not yet published.

[2] D. Knopf, Th. Wiedenhöfer, K. Lehrmann, F. Härtig, "A quantum of action on a scale? Dissemination of the quantum based kilogram", Metrologia 56 024003, 2019.

[3] M. Kochsiek, M. Gläser (eds.), "Comprehensive Mass Metrology”, ISBN 3-527-29614-X, p. 255, VCH, Berlin, 2000.

[4] DIN 12242-1, Laboratory glassware; interchangeable conical ground joints, dimensions, tolerances, 1980.

[5] PTB internal density determination by hydrostatic weighing at $20^{\circ} \mathrm{C}$, May 2020 .

[6] N. Greenwood, A. Earnshaw, Chemie der Elemente, Edition 1, VCH, Weinheim, ISBN 3527-26169-9, S. 1291, 1988.

[7] T. Ahrens et al., Mathematik, Ch. 11 Heidelberg, ISBN 978-3-8274-1758-9, 2008.

[8] www.wolfram.com/mathematica/ (2020-05-08). 